\title{
Crystal structure of pentasilver chloride silicate, $\mathrm{Ag}_{5}\left[\mathrm{SiO}_{4}\right] \mathrm{Cl}$
}

\author{
Wilhelm Klein ${ }^{1 . I I}$ and Martin Jansen*..I \\ 1 Technische Universität München, Forschungsneutronenquelle Heinz Maier-Leibnitz (FRM II), Lichtenbergstr. 1, 85747 Garching, Germany \\ II Max-Planck-Institut für Festkörperforschung, Heisenbergstr. 1, 70569 Stuttgart, Germany
}

Received March 13, 2009, accepted and available on-line April 1, 2009; CSD no. 409992

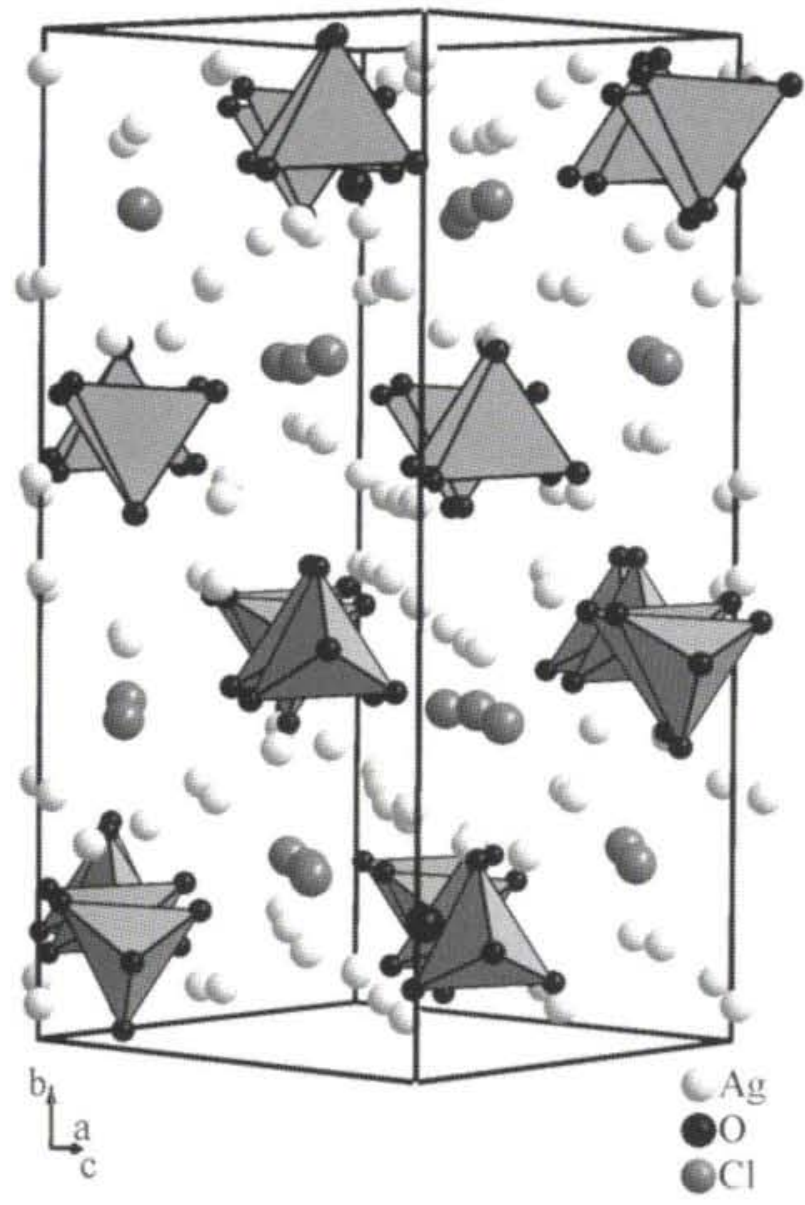

\begin{abstract}
$\mathrm{Ag}_{20} \mathrm{Cl}_{4} \mathrm{O}_{16} \mathrm{Si}_{4}$, monoclinic, $P 12{ }_{1} / c 1$ (no. 14),

$a=12.8931(8) \AA, b=19.092(1) \AA, c=12.7599(7) \AA$,

$\beta=118.356(1)^{\circ}, V=2764.0 \AA^{3}, Z=4, R_{g t}(F)=0.038$,

$w R_{\text {ref }}\left(F^{2}\right)=0.107, T=293 \mathrm{~K}$.
\end{abstract}

\section{Source of material}

Single crystals of $\mathrm{Ag}_{5} \mathrm{SiO}_{4} \mathrm{Cl}$ have been prepared via reaction of $\mathrm{AgCl}$ and elemental $\mathrm{Ag}$ and $\mathrm{Si}$ under elevated oxygen pressure using $1 \mathrm{ml} \mathrm{H}_{2} \mathrm{O}$ as an accelerator. The mixture was annealed for $60 \mathrm{~h}$ in gold crucibles placed in stainless steel autoclaves [1]. The reaction temperature and oxygen pressure were $823 \mathrm{~K}$ and $170 \mathrm{MPa}$, respectively.

\section{Discussion}

In the course of our systematic studies of ternary silver oxides, among others we have investigated the system $\mathrm{Ag}-\mathrm{Si}-\mathrm{O}$. Some interesting results have been obtained e.g. the first tetrasilicate [2], the subvalent (with respect to the silver part) $\mathrm{AgSSiO}_{4}$ [3], and recently the formally most simple orthosilicate, $\mathrm{Ag}_{4} \mathrm{SiO}_{4}$ [4]. At conditions suitable also for synthesis of the latter compound but in presence of a chlorine source [5] $\mathrm{Ag}_{5} \mathrm{SiO}_{4} \mathrm{Cl}$ has been obtained. The crystal structure is constituted of $\mathrm{Cl}$ atoms and isolated, slightly distorted $\mathrm{SiO}_{4}$ tetrahedra, all separated by $\mathrm{Ag}$ cations.

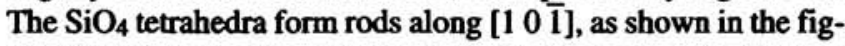
ure. All tetrahedra of a rod are oriented with one apex in the same direction so one threefold axis of all tetrahedra is nearly parallel to the $\left[\begin{array}{lll}1 & 0 & \overline{1}\end{array}\right]$ direction. The rods are packed in an almost hexagonal close manner, and every two rod layers perpendicular to the $b$ axis the tetrahedral orientation changes by $180^{\circ}$. Although both the unit cell parameters as well as the tetrahedral packing are close to trigonal symmetry, it has to be noted that the pseudotrigonal axes, i.e. the $b$-axis and the rod axis, are not parallel but perpendicular. $\mathrm{All} \mathrm{O}$ atoms are bound to one $\mathrm{Si}$ atom and two or three $\mathrm{Ag}$ atoms, while the $\mathrm{Cl}$ atoms are only coordinated to $\mathrm{Ag}$ with one or two short and four longer contacts. The Ag atoms are either in almost linear coordination by two $\mathrm{O}$ atoms or in trigonal planar by one $\mathrm{Cl}$ and two $\mathrm{O}$ atoms. The composition of the title compound resembles to the formerly obtained subvalent $\mathrm{Ag}_{5} \mathrm{SiO}_{4}$, where discrete $\mathrm{Ag}_{6}{ }^{4+}$ octahedra with $\mathrm{Ag}-\mathrm{Ag}$ contacts shorter than distances in elemental silver are found, besides $\mathrm{SiO}_{4}$ tetrahedra and isolated $\mathrm{Ag}$ cations in common coordination [3]. In the new compound, after formal addition of one $\mathrm{Cl}$ per formula to this composition, no short Ag-Ag contacts are observed. According to thermal analysis, $\mathrm{Ag}_{5} \mathrm{SiO}_{4} \mathrm{Cl}$ starts to decompose at $460^{\circ} \mathrm{C}$, slightly above $\mathrm{Ag}_{4} \mathrm{SiO}_{4}$ [4].

Table 1. Data collection and handling.

$\begin{array}{ll}\text { Crystal: } & \text { red block, size } 0.1 \times 0.1 \times 0.1 \mathrm{~mm} \\ \text { Wavelength: } & \text { Mo } K_{\alpha} \text { radiation }(0.71073 \AA) \\ \mu: & 144.24 \mathrm{~cm}^{-1} \\ \text { Diffractometer, scan mode: } & \text { SMART APEX, Bruker AXS, } \omega \\ 2 \theta_{\text {max: }} & 70.02^{\circ} \\ N(h k l)_{\text {measured, }} N(h k l)_{\text {hnique: }}: & 42905,6977 \\ \text { Criterion for } I_{\text {obs }}, N(h k l)_{\text {gt }}: & I_{\text {obs }}>2 \sigma\left(I_{\text {obs }}\right), 5654 \\ N(\text { param })_{\text {refined: }} & 398 \\ \text { Programs: } & \text { SHELXS-97 [6], Jana2006 [7], } \\ & \text { DLAMOND [8] }\end{array}$


Table 2. Atomic coordinates and displacement parameters (in $\AA^{2}$ ).

\begin{tabular}{|c|c|c|c|c|c|c|c|c|c|c|}
\hline Atom & Site & $x$ & $y$ & $z$ & $U_{11}$ & $U_{22}$ & $U_{33}$ & $U_{12}$ & $U_{13}$ & $U_{33}$ \\
\hline $\mathrm{Ag}(1)$ & $4 e$ & $0.07520(5)$ & $0.24815(2)$ & $0.41686(5)$ & $0.0294(3)$ & $0.0182(2)$ & $0.0292(3)$ & $-0.0034(2)$ & $0.0070(2)$ & $-0.0034(2)$ \\
\hline $\mathrm{Ag}(2)$ & $4 e$ & $0.70229(5)$ & $0.10752(3)$ & $0.04801(5)$ & $0.0200(2)$ & $0.0351(3)$ & $0.0231(2)$ & $-0.0012(2)$ & $0.0039(2)$ & $-0.0055(2)$ \\
\hline $\mathbf{A g}(3)$ & $4 e$ & $0.69287(5)$ & $0.03756(3)$ & $0.80237(5)$ & $0.0339(3)$ & $0.0261(2)$ & $0.0235(2)$ & $0.0047(2)$ & $0.0147(2)$ & $0.0067(2)$ \\
\hline $\mathrm{Ag}(4)$ & $4 e$ & $0.56725(5)$ & $0.24826(3)$ & $0.42946(5)$ & $0.0318(3)$ & $0.0230(3)$ & $0.0323(3)$ & $0.0066(2)$ & $0.0098(2)$ & $00066(2)$ \\
\hline $\operatorname{Ag}(5)$ & $4 e$ & $0.19344(4)$ & $0.10033(3)$ & $0.55641(4)$ & $0.0218(3)$ & $0.0306(3)$ & $0.0204(2)$ & $0.0041(2)$ & $0.0015(2)$ & $00001(2)$ \\
\hline$A g(6)$ & $4 e$ & $0.44021(4)$ & $0.10729(3)$ & $0.29353(5)$ & $0.0184(2)$ & $0.0320(3)$ & $0.0215(2)$ & $0.0034(2)$ & $0.0029(2)$ & $-0,0001(2)$ \\
\hline $\mathbf{A g}(7)$ & $4 e$ & $0.94141(4)$ & $0.10012(3)$ & $0.79519(5)$ & $0.0219(3)$ & $0.0303(3)$ & $0.0233(2)$ & $0.0006(2)$ & $0.0037(2)$ & $-0.0050(2)$ \\
\hline Ag(8) & $4 e$ & $0.94021(5)$ & $0.03908(3)$ & $0.05765(5)$ & $0.0256(3)$ & $0.0253(2)$ & $0.0315(3)$ & $-0.0051(2)$ & $0.0150(2)$ & $-0.0053(2)$ \\
\hline Ag(9) & $4 e$ & $0.94393(5)$ & $0.04021(3)$ & $0.55823(4)$ & $0.0326(3)$ & $0.0256(2)$ & $0.0236(2)$ & $0.0062(2)$ & $0.0163(2)$ & $00063(2)$ \\
\hline $\mathbf{A g}(10)$ & $4 e$ & $0.19112(5)$ & $0.04614(3)$ & $0.81431(5)$ & $0.0240(3)$ & $0.0310(3)$ & $0.0305(3)$ & $-0.0100(2)$ & $0.0133(2)$ & $-0.0049(2)$ \\
\hline $\mathrm{Ag}(11)$ & $4 e$ & $0.43317(7)$ & $0.03980(4)$ & $0.06113(7)$ & $0.0497(4)$ & $0.0526(4)$ & $0.0754(5)$ & $0.0247(3)$ & $0.0408(4)$ & $0.0435(4)$ \\
\hline $\mathrm{Ag}(12)$ & $4 e$ & $0.19664(5)$ & $0.05639(3)$ & $0.32915(5)$ & $0.0416(3)$ & $0.0273(3)$ & $0.0365(3)$ & $0.0076(2)$ & $0.0227(3)$ & $00100(2)$ \\
\hline $\mathbf{A g}(13)$ & $4 e$ & $0.54006(7)$ & $0.19796(3)$ & $0.68326(7)$ & $0.0650(5)$ & $0.0288(3)$ & $0.0673(5)$ & $-0.0029(3)$ & $0.0512(4)$ & $-0,0081(3)$ \\
\hline Ag(14) & $4 e$ & $0.79953(6)$ & $0.24740(3)$ & $0.72487(6)$ & $0.0462(4)$ & $0.0264(3)$ & $0.0353(3)$ & $-0.0084(2)$ & $0.0168(3)$ & $-00026(2)$ \\
\hline $\operatorname{Ag}(15)$ & $4 e$ & $0.33231(7)$ & $0.20334(3)$ & $0.99470(7)$ & $0.0580(4)$ & $0.0236(3)$ & $0.0600(4)$ & $0.0038(2)$ & $0.0424(4)$ & $00065(2)$ \\
\hline $\mathrm{Ag}(16)$ & $4 e$ & $0.26037(6)$ & $0.24600(3)$ & $0.70911(6)$ & $0.0347(3)$ & $0.0278(3)$ & $0.0567(4)$ & $0.0030(2)$ & $0.0155(3)$ & $00103(3)$ \\
\hline $\operatorname{Ag}(17)$ & $4 e$ & $0.82841(8)$ & $0.19326(3)$ & $0.46273(8)$ & $0.0745(5)$ & $0.0273(3)$ & $0.0747(5)$ & $0.0064(3)$ & $0.0580(4)$ & $0.0014(3)$ \\
\hline $\operatorname{Ag}(18)$ & $4 e$ & $0.99868(7)$ & $0.19730(3)$ & $0.14585(7)$ & $0.0520(4)$ & $0.0265(3)$ & $0.0687(5)$ & $-0.0024(2)$ & $0.0404(4)$ & $00029(3)$ \\
\hline $\operatorname{Ag}(19)$ & $4 e$ & $0.67068(6)$ & $0.05271(3)$ & $0.28340(6)$ & $0.0399(3)$ & $0.0266(3)$ & $0.0505(4)$ & $-0.0098(2)$ & $0.0296(3)$ & $-00068(2)$ \\
\hline $\operatorname{Ag}(20)$ & $4 e$ & $0.43063(8)$ & $0.04573(4)$ & $0.55860(7)$ & $0.0751(5)$ & $0.0543(4)$ & $0.0725(5)$ & $-0.0448(4)$ & $0.0560(5)$ & $-00338(4)$ \\
\hline $\operatorname{Si}(1)$ & $4 e$ & $0.6789(1)$ & $0.10260(8)$ & $0.5654(1)$ & $0.0124(7)$ & $0.0166(7)$ & $0.0121(7)$ & $0.0012(5)$ & $0.0048(6)$ & $00007(5)$ \\
\hline$O(11)$ & $4 e$ & $0.6000(4)$ & $0.0676(2)$ & $0.4342(4)$ & $0.022(2)$ & $0.026(2)$ & $0.015(2)$ & $0.004(2)$ & $0.006(2)$ & $-0001(2)$ \\
\hline$O(12)$ & $4 e$ & $0.6070(4)$ & $0.1000(2)$ & $0.6412(4)$ & $0.025(2)$ & $0.022(2)$ & $0.021(2)$ & $0.002(2)$ & $0.016(2)$ & $0002(2)$ \\
\hline$O(13)$ & $4 e$ & $0.8015(4)$ & $0.0587(2)$ & $0.6360(4)$ & $0.023(2)$ & $0.017(2)$ & $0.023(2)$ & $0.006(2)$ & $0.010(2)$ & $0002(2)$ \\
\hline O(14) & $4 e$ & $0.7082(4)$ & $0.1843(2)$ & $0.5519(4)$ & $0.027(2)$ & $0.015(2)$ & $0.022(2)$ & $-0.001(2)$ & $0.010(2)$ & $0002(2)$ \\
\hline $\operatorname{Si}(2)$ & $4 e$ & $0.4250(1)$ & $0.10725(8)$ & $0.8229(1)$ & $0.0131(7)$ & $0.0139(7)$ & $0.0161(7)$ & $0.0001(5)$ & $0.0057(6)$ & $00012(5)$ \\
\hline$O(21)$ & $4 e$ & $0.5525(4)$ & $0.0698(2)$ & $0.8934(4)$ & $0.021(2)$ & $0.026(2)$ & $0.031(2)$ & $0.004(2)$ & $0.009(2)$ & $-0.004(2)$ \\
\hline $\mathbf{O}(22)$ & $4 e$ & $0.3496(4)$ & $0.0624(2)$ & $0.6997(4)$ & $0.022(2)$ & $0.016(2)$ & $0.017(2)$ & $0.003(2)$ & $0.002(2)$ & $-0001(2)$ \\
\hline$O(23)$ & $4 e$ & $0.3543(4)$ & $0.1062(2)$ & $0.9005(4)$ & $0.022(2)$ & $0.024(2)$ & $0.024(2)$ & $0.000(2)$ & $0.014(2)$ & $0001(2)$ \\
\hline$O(24)$ & $4 e$ & $0.4393(4)$ & $0.1882(2)$ & $0.7917(4)$ & $0.022(2)$ & $0.016(2)$ & $0.025(2)$ & $-0.001(2)$ & $0.009(2)$ & $0001(2)$ \\
\hline $\operatorname{Si}(3)$ & $4 e$ & $0.9332(1)$ & $0.10047(8)$ & $0.3138(1)$ & $0.0125(7)$ & $0.0125(7)$ & $0.0130(7)$ & $-0.0011(5)$ & $0.0037(6)$ & $-0,0004(5)$ \\
\hline$O(31)$ & $4 e$ & $1.0606(4)$ & $0.1385(2)$ & $0.3915(4)$ & $0.014(2)$ & $0.020(2)$ & $0.021(2)$ & $-0.003(2)$ & $0.002(2)$ & $-0.000(2)$ \\
\hline$O(32)$ & $4 e$ & $0.8620(4)$ & $0.0957(2)$ & $0.3898(4)$ & $0.020(2)$ & $0.022(2)$ & $0.025(2)$ & $0.001(2)$ & $0.014(2)$ & $0002(2)$ \\
\hline$O(33)$ & $4 e$ & $0.8604(4)$ & $0.1466(2)$ & $0.1937(4)$ & $0.014(2)$ & $0.022(2)$ & $0.019(2)$ & $-0.001(2)$ & $0.004(2)$ & $-0.001(2)$ \\
\hline$O(34)$ & $4 e$ & $0.9582(4)$ & $0.0220(2)$ & $0.2791(4)$ & $0.018(2)$ & $0.020(2)$ & $0.018(2)$ & $0.002(2)$ & $0.008(2)$ & $-0,002(2)$ \\
\hline $\mathrm{Si}(4)$ & $4 e$ & $0.1739(1)$ & $0.10460(8)$ & $0.0656(1)$ & $0.0165(8)$ & $0.0133(7)$ & $0.0150(7)$ & $0.0005(5)$ & $0.0066(6)$ & $0.0011(5)$ \\
\hline$O(41)$ & $4 e$ & $0.2934(3)$ & $0.1509(2)$ & $0.1403(4)$ & $0.012(2)$ & $0.027(2)$ & $0.014(2)$ & $-0.008(2)$ & $-0.001(2)$ & $-0004(2)$ \\
\hline$O(42)$ & $4 e$ & $0.0971(4)$ & $0.1429(2)$ & $-0.0630(4)$ & $0.019(2)$ & $0.018(2)$ & $0.018(2)$ & $-0.003(2)$ & $0.002(2)$ & $-0.003(2)$ \\
\hline$O(43)$ & $4 e$ & $0.0990(4)$ & $0.0998(2)$ & $0.1395(4)$ & $0.020(2)$ & $0.027(2)$ & $0.025(2)$ & $-0.003(2)$ & $0.014(2)$ & $-0.006(2)$ \\
\hline$O(44)$ & $4 e$ & $0.2100(4)$ & $0.0262(2)$ & $0.0418(4)$ & $0.029(2)$ & $0.019(2)$ & $0.025(2)$ & $0.001(2)$ & $0.015(2)$ & $-0.003(2)$ \\
\hline $\mathrm{Cl}(1)$ & $4 e$ & $0.3358(2)$ & $0.17290(8)$ & $0.4338(2)$ & $0.0281(8)$ & $0.0230(7)$ & $0.0335(8)$ & $-0.0032(6)$ & $0.0149(7)$ & $-00042(6)$ \\
\hline $\mathrm{Cl}(2)$ & $4 e$ & $0.0735(2)$ & $0.18130(9)$ & $0.6708(2)$ & $0.0309(9)$ & $0.0263(7)$ & $0.0324(8)$ & $0.0015(6)$ & $0.0147(7)$ & $00005(6)$ \\
\hline $\mathrm{Cl}(3)$ & $4 e$ & $0.5788(1)$ & $0.17058(9)$ & $0.1727(2)$ & $0.0243(8)$ & $0.0222(7)$ & $0.0420(9)$ & $-0.0003(6)$ & $0.0148(7)$ & $00009(6)$ \\
\hline $\mathrm{Cl}(4)$ & $4 e$ & $0.8077(2)$ & $0.17905(9)$ & $0.9026(2)$ & $0.042(1)$ & $0.0254(7)$ & $0.0290(8)$ & $-0.0028(7)$ & $0.0168(7)$ & $-00007(6)$ \\
\hline
\end{tabular}

Acknowledgments. We gratefully acknowledge E.-M. Peters for performing the EDX analyses and Dr. J. Nuss for recording the $\mathrm{X}$-ray single crystal diffraction data.

\section{References}

1. Linke, C.; Jansen, $\mathrm{M}$.: Über $\mathrm{Ag}_{2} \mathrm{SnO}_{3}$, das erste Silberstannat. Z. Anorg. Allg. Chem. 623 (1997) 1441-1446.

2. Jansen, M.; Keller, H.-L.: $\mathrm{Ag}_{10} \mathrm{Si}_{4} \mathrm{O}_{13}$, das erste Tetrasilicat. Angew. Chem. 91 (1979) 500; Angew. Chem. Int. Ed. Engl. 18 (1979) 464.

3. Linke, C.: Jansen, M.: Subvalent Ternary Silver Oxides: Synthesis, Structural Characterization, and Physical Properties of Pentasilver Orthosilicate, $\mathrm{AgsSiO}_{4}$. Inorg. Chem. 33 (1994) 2614-2616.

4. Klein, W.; Jansen, M.: Synthesis and Crystal Structure of Silver Nesosilicate, Ag4SiO4. Z. Anorg. Allg. Chem. 634 (2008) 1077-1081.
5. Curda, J.; Peters, E.-M.; Klein, W.; Jansen, M.: Crystal structure of silver chloride chromate(VI), $\mathrm{Ag}_{3} \mathrm{ClCrO}_{4}$. Z. Kristallogr. NCS 216 (2001) 180.

6. Sheldrick, G. M.: SHELXS-97. Program for the Solution of Crystal Structures. University of Göttingen, Germany 1997.

7. Petricek, V.; Dusek, M.; Palatinus L.: Jana2006. Structure Determination Software Programs. Institute of Physics, Praha, Czech Republit.

8. Brandenburg, K.: DIAMOND. Visual Crystal Structure Information System. Version 3.1e. Crystal Impact, Bonn, Germany 1998. 